\title{
ASYMPTOTIC EQUIPARTITION OF KINETIC AND STRAIN ENERGY FOR ELASTIC WAVES IN ANISOTROPIC MEDIA*
}

\author{
BY \\ GEORGE DASSIOS AND EFSTRATIOS GALANIS \\ National Technical University of Athens
}

1. Introduction. In wave motion the term "equipartition of energy" is used to denote the equality of kinetic and potential energy. Equipartition can be attained in finite or in infinite time (asymptotic equipartition).

The first result on equipartition of energy, due to Lax and Phillips [8], is actually an asymptotic result. Duffin [5] has proved that equipartition is attained in finite time as long as the Cauchy data have compact support. One of the authors [2] has extended these results to electromagnetic waves, proving that if the Cauchy data are in $L^{2}\left(\mathbb{R}^{3}\right)$ then asymptotic equipartition of the electric and the magnetic energy results, and that this equipartition is attained in finite time, whenever the initial disturbance is confined to a compact set. The same author [3] has proved that for linear elastic wave propagation in homogeneous and isotropic media there is no transfer of energy from the longitudinal to shear wave and vice versa. Hence the energy that is stored initially in each type of wave yields a constant of the motion. Combining this result with that of Duffin, it follows that for initial data with compact support the kinetic and strain energy of the fast $(P)$ wave is equipartitioned first and the same thing happens to the slow $(S)$ wave at a later time. The asymptotic form of this result also remains true. Partition of energy for uniformly propagative systems was shown by Costa [1]. Results on equipartition of energy for abstract wave equations were established by Goldstein [6, 7], Levine [9], and others.

In this paper we investigate the problem of equipartition of energy for elastic waves that propagate in a homogeneous but anisotropic medium. As is well known, in this case there are three waves propagating in any direction which are arbitrarily polarized. By generalizing the Riemann-Lebesgue lemma of harmonic analysis we are able to show asymptotic equipartition of kinetic and strain energy for each one of the three types of elastic waves that exist in an anisotropic medium. For general information on wave propagation in linear elastic homogeneous and anisotropic media, see Duff [4], Musgrave [10], and Synge [11, 12].

2. The elastic wave equation. We consider a homogeneous linear hyperelastic material filling up the space $\mathbb{R}^{3}$ with constitutive equation

$$
\tau_{i j}=c_{i j k l} e_{k l}
$$

* Received April 26, 1979. 
where $\tau_{i j}$ is the stress tensor, $c_{i j k l}$ are the elasticities and $e_{k l}$ is the strain tensor. Here and throughout summation from 1 to 3 is understood whenever a repeated index appears. We have the symmetry conditions

$$
c_{i j k l}=c_{j i k l}=c_{k l i j}
$$

which reduce the number of different elasticities to 21 . The strain-energy density function is given by

$$
W=\frac{1}{2} c_{i j k l} e_{i j} e_{k l} .
$$

In the absence of body forces the equations of motion take the form

$$
\rho \ddot{u}_{i}=\tau_{i j, j}, \quad i=1,2,3
$$

or

$$
\ddot{u}_{i}-\frac{1}{\rho} c_{i j k l} u_{k, l j}=0, \quad i=1,2,3,
$$

where $\rho$ is the mass density and $\mathbf{u}=\left(u_{1}, u_{2}, u_{3}\right)$ the displacement field.

It is well known that if the initial data satisfy the conditions

$$
\mathbf{u}(\mathbf{x}, 0)=\mathbf{u}_{0} \in\left[H^{1}\left(\mathbb{R}^{3}\right)\right]^{3}, \quad \dot{\mathbf{u}}(\mathbf{x}, 0)=\mathbf{u}_{1} \in\left[L^{2}\left(\mathbb{R}^{3}\right)\right]^{3},
$$

where $H^{1}$ is the Sobolev space $W^{1,2}$ (the space of square-integrable functions whose gradient is also square-integrable), then there exists a unique solution $\mathbf{u}(\mathbf{x}, t)$ such that for any $t \geq 0$,

$$
\mathbf{u}(\mathbf{x}, t) \in\left[H^{1}\left(\mathbb{R}^{3}\right)\right]^{3} \quad \text { and } \quad \dot{\mathbf{u}}(\mathbf{x}, t) \in\left[L^{2}\left(\mathbb{R}^{3}\right)\right]^{3}
$$

and

$$
E(t)=E(0), \quad t \geq 0
$$

where $E(t)$, the energy at time $t$, is the sum of the kinetic energy

$$
K(t)=\frac{1}{2} \int_{\mathbb{B}^{3}} \rho \dot{u}_{i} \dot{u}_{i} d x
$$

and the strain energy

$$
P(t)=\frac{1}{2} \int_{\mathbb{R} 3} c_{i j k l} u_{i, j} u_{k, l} d x .
$$

Moreover, if the initial data are $C^{\infty}$-functions with compact support in $\mathbb{R}^{3}$, then the solution is also a $C^{\infty}$-function and has, for each $t \geq 0$, compact support in $\mathbb{R}^{3}$.

3. Spatial harmonic decomposition. Consider the Fourier transform of the displacement vector $\mathbf{u}$ in space:

$$
\hat{\mathbf{u}}(\xi, t)=(2 \pi)^{-3 / 2} \int_{\mathbb{R}^{3}} e^{i \xi \cdot \mathbf{x}} \mathbf{u}(\mathbf{x}, t) d t .
$$

Since

$$
\hat{u}_{k, l}(\xi, t)=-i \xi_{l} \hat{u}_{k}(\xi, t),
$$


the system of equations (5) becomes

$$
\frac{d^{2}}{d t^{2}}\left[\rho \hat{u}_{i}(\xi, t)\right]=-r^{2} c_{i j k l} \alpha_{j} \alpha_{l} \hat{u}_{k}(\xi, t)
$$

where

$$
\xi=r \alpha=r\left(\alpha_{1}, \alpha_{2}, \alpha_{3}\right), \quad r=|\xi|, \quad|\alpha|=1 .
$$

The system of ordinary differential equations (11) is written in dyadic form as

$$
\frac{d^{2}}{d t^{2}} \hat{\mathbf{u}}(t)+r^{2} \mathbb{B} \cdot \hat{\mathbf{u}}(t)=0
$$

where the acoustic tensor $\mathbb{B}$ is given by

$$
B_{i k}=c_{i j k l} \alpha_{j} \alpha_{l} .
$$

The positive definiteness of the strain energy density function implies that all three eigenvalues of $\mathbb{B}$ are positive and therefore their positive square roots are real and nonnegative. The dyadic $\mathbb{B}$ takes the following normal form:

$$
\mathbb{B}(\boldsymbol{\alpha})=\sum_{i=1}^{3} v_{i}^{2}(\boldsymbol{\alpha}) \mathbf{e}_{i}(\boldsymbol{\alpha}) \otimes \mathbf{e}_{i}(\boldsymbol{\alpha})
$$

where $v_{i}^{2}(\boldsymbol{\alpha})$ is the eigenvalue that corresponds to the eigenvector $\mathbf{e}_{i}(\boldsymbol{\alpha})$ associated with the particular direction $\alpha$. Without loss of generality we assume that

$$
v_{1} \geq v_{2} \geq v_{3} .
$$

For each direction $\alpha$ the unit vectors $\mathbf{e}_{i}, i=1,2,3$, form an orthogonal set. In the particular direction $\alpha$ three different waves propagate with phase velocities $v_{i}(\alpha)$ and polarization vectors $\mathbf{e}_{i}(\boldsymbol{\alpha})$. In general, no polarization vector is parallel to the direction of propagation. Obviously

$$
\mathbb{B}(\alpha)=\mathbb{A}^{2}(\alpha)
$$

where

$$
\mathbb{A}(\boldsymbol{\alpha})=\sum_{i=1}^{3} v_{i}(\boldsymbol{\alpha}) \mathbf{e}_{i}(\boldsymbol{\alpha}) \otimes \mathbf{e}_{i}(\boldsymbol{\alpha}) .
$$

We next prove the following lemma.

Lemma 1. The solution of the equation

$$
\frac{d^{2}}{d t^{2}} \hat{\mathbf{u}}(t)+(r \mathbb{A})^{2} \hat{\mathbf{u}}(t)=0
$$

that satisfies the initial conditions

$$
\begin{aligned}
\hat{\mathbf{u}}(0) & =[\mathbf{u}(\mathbf{x}, 0)]^{\wedge}=\hat{\mathbf{u}}_{0}(\xi), \\
\left.\frac{d}{d t} \mathbf{u}(t)\right|_{t=0} & =\left[\left.\frac{d}{d t} \mathbf{u}(x, t)\right|_{t=0}\right]^{\wedge}=\hat{\mathbf{u}}_{1}(\xi)
\end{aligned}
$$


is given by

$$
\hat{\mathbf{u}}(t)=\sum_{i=1}^{3} \mathbf{e}_{i} \otimes \mathbf{e}_{i} \cdot\left[\hat{\mathbf{u}}_{0} \cos \left(\operatorname{tr} v_{i}\right)+\hat{\mathbf{u}}_{1} \frac{\sin \left(\operatorname{tr} v_{i}\right)}{r v_{i}}\right]
$$

Proof. Solving (19) formally, we obtain

$$
\hat{\mathbf{u}}(t)=\cos (\operatorname{tr} A) \cdot \hat{\mathbf{u}}_{0}+\frac{1}{r} \sin (\operatorname{tr} A) \cdot\left[A^{-1} \cdot \hat{\mathbf{u}}_{1}\right]
$$

Using standard identities of the calculus of dyadics one obtains

$$
\begin{aligned}
\cos (\operatorname{tr} A) & =\sum_{n=0}^{\infty}(-1)^{n} \frac{(\operatorname{tr})^{2 n}}{(2 n) !} A^{2 n} \\
& =\sum_{i=1}^{3}\left[\sum_{n=0}^{\infty}(-1)^{n} \frac{\left(\operatorname{tr} v_{i}\right)^{2 n}}{(2 n) !}\right] \mathbf{e}_{i} \otimes \mathbf{e}_{i} \\
& =\sum_{i=1}^{3} \mathbf{e}_{i} \otimes \mathbf{e}_{i} \cos \left(\operatorname{tr} v_{i}\right) .
\end{aligned}
$$

Similarly

$$
\sin (\operatorname{tr} A)=\sum_{i=1}^{3} \mathbf{e}_{i} \otimes \mathbf{e}_{i} \sin \left(\operatorname{tr} v_{i}\right)
$$

Also

$$
A^{-1}=\sum_{i=1}^{3} \frac{1}{v_{i}} \mathbf{e}_{i} \otimes \mathbf{e}_{i}
$$

Substituting (24), (25) and (26) into (23) and performing the indicated contractions of the dyadics, one arrives at the form (22) for the solution of (19). This completes the proof.

We next discuss the properties of the solution $\hat{\mathbf{u}}(t)$ as given by (22). Each one of the time-independent constants in (22), i.e. $\hat{\mathbf{u}}_{0}, \hat{\mathbf{u}}_{1}, v_{i}$ and $\mathbf{e}_{i}$, are functions of the direction $\boldsymbol{\alpha}$. For a fixed direction $\boldsymbol{\alpha}, \hat{\mathbf{u}}(t)$ describes the propagation of three plane waves. These are

$$
\mathbf{w}_{i}=\mathbf{e}_{i} \otimes \mathbf{e}_{i} \cdot\left[\hat{\mathbf{u}}_{1} \cos \left(\operatorname{tr} v_{i}\right)+\hat{\mathbf{u}}_{1} \frac{\sin \left(\operatorname{tr} v_{i}\right)}{r v_{i}}\right]
$$

for $i=1,2,3$. Each one of the $\mathbf{w}_{i}$ s propagates in the direction $\alpha$ with different phase velocity and polarization vector. The three polarization vectors are mutually orthogonal but in general, none of them points in the direction of propagation. According to our assumption (16), in each direction $\alpha$ the first wave $w_{1}$ is the fastest and the third wave $w_{3}$ is the slowest. The displacement field $\mathbf{u}(\mathbf{x}, t)$, is not, in general, a plane wave; therefore the three polarization vectors corresponding to the three phase velocities associated with the direction $\mathbf{x}$ are no longer orthogonal.

The space-time characteristic cone (see [4] for details) emanating from a point $(\mathbf{x}, t)$ has three noncircular conical sheets corresponding to the three phase velocities. For their geometric properties see [10]-[12]. The wave surface, defined as the intersection of the wave cone with the $t$-constant plane, consists of three nonspherical sheets. The geometry of the wave surface can be very complicated. Any half-line from $\mathbf{x}$ will meet the wave surface at most 75 times. The wave surface is symmetric with respect to the source point $\mathbf{x}$. 
It was shown by Duff [4] that if the initial data are restricted in a neighborhood of the origin of diameter $\delta$, then there will be three "sharp waves" traveling with velocities $v_{1}$, $v_{2}$ and $v_{3}$ along the three wave sheets. Each sharp wave shell will have a thickness $\delta$. In addition to the sharp wave, there will also be a "continuous wave" that is supported in the region defined by the intersection of the convex hull of the outermost (fastest) wave sheet and the exterior of the innermost (slowest) wave sheet. Therefore an observer outside the initial support of the disturbance will first feel either the first sharp wave or the continuous wave, depending on whether the outermost wave sheet is convex or not. Then he will experience a finite succession of sharp and continuous waves and finally the disturbance will terminate with the last sharp wave. The interior of the innermost wave sheet is a lacuna.

4. Asymptotic equipartition of energy. We begin with a generalization of the Riemann-Lebesgue lemma.

Lemma 2. Assume that the function $f: \mathbb{R}^{3} \rightarrow \mathbb{R}$ is an absolutely integrable function and that $v: S_{3} \rightarrow \mathbb{R}$ is a continuous function defined on the unit sphere $S_{3}=\left\{\alpha \in \mathbb{R}^{3}|| \alpha \mid=1\right\}$ satisfying the relation

$$
0<v_{0} \leq v(\boldsymbol{\alpha})
$$

for each $\alpha \in S_{3}$. Then

$$
\lim _{t \rightarrow+\infty} I(t)=\lim _{t \rightarrow+\infty} \int_{\mathbb{R}^{3}}|f(\mathbf{x})| e^{i t|\mathbf{x}| v(\alpha)} d^{3} x=0 .
$$

(Note that if $v(\alpha)=$ constant then the above lemma reduces to the Riemann-Lebesgue lemma of harmonic analysis.)

Proof. Introduce polar coordinates

$$
r=|\mathbf{x}|, \quad \alpha=\mathbf{x} /|\mathbf{x}| .
$$

Then in the light of Fubini's theorem the integral can be written as

$$
I(t)=\int_{S_{3}} \int_{0}^{\infty}\left|f_{p}(r, \alpha)\right| e^{i \operatorname{tr} v(\alpha)} r^{2} d r d^{2} \alpha,
$$

where $f_{p}(r, \alpha)=f(\mathbf{x})$ and $d^{2} \alpha$ represents the differential solid angle. Define

$$
F(t, \alpha)=\int_{0}^{\infty}\left|f_{p}(r, \alpha)\right| e^{i \operatorname{tr} v(\alpha)} r^{2} d r ;
$$

then

$$
I(t)=\int_{S_{3}} F(t, \alpha) d^{2} \alpha .
$$

Take any sequence $\left\{t_{n}\right\}_{n=1}^{\infty}$ such that $t_{n} \rightarrow+\infty$ as $n \rightarrow+\infty$. Then for each $n$

$$
\left|F\left(t_{n}, \alpha\right)\right| \leq \int_{0}^{\infty}\left|f_{p}(r, \alpha)\right| r^{2} d r=g(\alpha)
$$

and

$$
\int_{S_{3}} g(\alpha) d^{2} \alpha=\int_{\mathbb{R}^{3}}|f(\mathbf{x})| d^{3} x<+\infty .
$$


The ordinary Riemann-Lebesgue lemma gives

$$
\lim _{n \rightarrow \infty} F\left(t_{n}, \alpha\right)=\lim _{n \rightarrow \infty} \int_{0}^{\infty}\left|f_{p}(r, \alpha)\right| e^{i t_{n} r v(\alpha)} r^{2} d r=0,
$$

and by hypothesis (28), this is true for every $\alpha \in S_{3}$. By (34), (35), (36) and the Lebesgue dominated convergence theorem one obtains

$$
\lim _{n \rightarrow+\infty} I\left(t_{n}\right)=\int_{S_{3}} \lim _{n \rightarrow+\infty} F\left(t_{n}, \alpha\right) d^{2} \alpha=0
$$

which in turn implies that

$$
\lim _{t \rightarrow+\infty} I(t)=0
$$

This completes the proof of the lemma.

Our assumption (16) implies that the three wave sheets may touch but not cross one other, i.e. the wave sheet that corresponds to the second phase velocity lies always between the outermost and the innermost wave sheet.

Define the $n$-wave $\mathbf{u}^{n}$ by

$$
\mathbf{u}^{n}(\mathbf{x}, t)=\left[\mathbf{w}_{n}(\xi, t)\right]^{\vee}(\mathbf{x}, t)
$$

for each $n=1,2,3$, where $\mathbf{w}_{n}$ is given by (27) and ${ }^{\vee}$ indicates the inverse Fourier transform operator. Then

$$
\mathbf{u}(\mathbf{x}, t)=\sum_{n=1}^{3} \mathbf{u}^{n}(\mathbf{x}, t)
$$

We also define the $n$-kinetic energy by

$$
K^{n}(t)=\frac{1}{2} \int_{\mathbb{R} 3} \rho\left|\dot{\mathbf{u}}^{n}\right|^{2} d^{3} x
$$

and the $n$-strain energy by

$$
S^{n}(t)=\frac{1}{2} \int_{\mathbb{R}^{3}} c_{i j k l} u_{i, j}^{n} u_{k, l}^{n} d^{3} x
$$

for each $n=1,2,3$. The n-energy is defined by

$$
E^{n}(t)=K^{n}(t)+S^{n}(t)
$$

for $n=1,2,3$.

We state now and prove the basic equipartition theorem.

THEOREM. Let $\mathbf{u}(\mathbf{x}, t)$ be a solution of the system

$$
\ddot{u}_{i}-\frac{1}{\rho} c_{i j k l} u_{k, l j}=0, \quad i=1,2,3
$$

in $C^{2}\left(\mathbb{P}^{3} \times[0,+\infty)\right)$, satisfying the initial conditions

$$
\mathbf{u}(\mathbf{x}, 0)=\mathbf{u}_{0}(\mathbf{x}), \quad \dot{\mathbf{u}}(\mathbf{x}, 0)=\mathbf{u}_{1}(\mathbf{x})
$$


where $\mathbf{u}_{0} \in\left[H^{1}\left(\mathbb{R}^{3}\right)\right]^{3}, \mathbf{u}_{1} \in\left[L^{2}\left(\mathbb{R}^{3}\right)\right]^{3}$. Let $v_{i}(\alpha), i=1,2,3$, be the eigenvalues of the tensor

$$
B_{i k}=c_{i j k l} \alpha_{j} \alpha_{l}
$$

relative to the direction $\alpha$, and assume that

$$
\inf \left\{v_{i}(\alpha) \mid i=1,2,3, \alpha \in S_{3}\right\} \geq v_{0}>0 .
$$

Then for each $n=1,2,3$ we have

$$
\lim _{t \rightarrow \infty} K^{n}(t)=\lim _{t \rightarrow \infty} S^{n}(t)=\frac{1}{2} E^{n}(0)
$$

and therefore

$$
\lim _{t \rightarrow \infty} K(t)=\lim _{t \rightarrow \infty} S(t)=\frac{1}{2} E(0),
$$

where $K^{n}, S^{n}, E^{n}$ are the kinetic, the strain and the total energy of the $n$-wave respectively, and $K, S, E$ are the corresponding energies of the elastic wave.

Proof. By Parseval's theorem we have

$$
K(t)=\frac{1}{2} \int_{\mathbb{B}^{3}} \rho|\dot{\mathbf{u}}|^{2} d^{3} x=\frac{1}{2} \int_{\mathbb{R}^{3}} \rho|\hat{\mathbf{u}}|^{2} d^{3} \xi
$$

and

$$
\begin{aligned}
S(t) & =\frac{1}{2} \int_{\mathbb{B}^{3}} c_{i j k l} u_{i, j} u_{k, l} d^{3} x \\
& =\frac{1}{2} \int_{\mathbb{R}^{3}} c_{i j k l}\left(-i \xi_{j} \hat{u}_{i}\right)\left(i \xi_{l} \hat{u}_{k}^{*}\right) d^{3} \xi \\
& =\frac{1}{2} \int_{\mathbb{P}^{3}} r^{2}(\hat{\mathbf{u}}) \cdot \mathbb{B} \cdot(\hat{\mathbf{u}})^{*} d^{3} \xi \\
& =\frac{1}{2} \sum_{n=1}^{3} \int_{\mathbb{R}^{3}} r^{2} v_{n}^{2}\left|\mathbf{e}_{n} \cdot \hat{\mathbf{u}}\right|^{2} d^{3} \xi \\
& =\frac{1}{2} \sum_{n=1}^{3} \int_{\mathbb{R}^{3}} r^{2} v_{n}^{2}\left|\mathbf{w}_{n}\right|^{2} d^{3} \xi .
\end{aligned}
$$

Similarly,

$$
K^{n}(t)=\frac{1}{2} \int_{\mathbb{R}^{3}} \rho\left|\mathbf{w}_{n}\right|^{2} d^{3} \xi, \quad n=1,2,3
$$

and

$$
S^{n}(t)=\frac{1}{2} \int_{\mathbb{R}^{3}} r^{2} v_{i}^{2}\left|\mathbf{w}_{n}\right|^{2} d^{3} \xi, \quad n=1,2,3 .
$$

Clearly

$$
K(t)=\sum_{n=1}^{3} K^{n}(t)
$$


and

$$
S(t)=\sum_{n=1}^{3} S^{n}(t)
$$

Using the forms (27) we obtain for each $n=1,2,3$

$$
\begin{aligned}
K^{n}(t)= & \frac{1}{4} \int_{\mathbb{R}^{3}}\left[\left|\mathbf{e}_{n} \cdot \hat{\mathbf{u}}_{1}\right|^{2}+r^{2} v_{n}^{2}\left|\mathbf{e}_{n} \cdot \hat{\mathbf{u}}_{0}\right|^{2}\right] d^{3} \xi \\
& +\frac{1}{4} \int_{\mathbb{R}^{3}}\left[\left|\mathbf{e}_{n} \cdot \hat{\mathbf{u}}_{1}\right|^{2}-r^{2} v_{n}^{2}\left|\mathbf{e}_{n} \cdot \hat{\mathbf{u}}_{0}\right|^{2}\right] \cos \left(2 \operatorname{tr} v_{n}\right) d^{3} \xi \\
& -\frac{1}{2} \int_{\mathbb{R}^{3}} \operatorname{Re}\left[r v_{n}\left(\mathbf{e}_{n} \cdot \hat{\mathbf{u}}_{0}\right)\left(\mathbf{e}_{n} \cdot \hat{\mathbf{u}}_{1}\right)^{*}\right] \sin \left(2 \operatorname{tr} v_{n}\right) d^{3} \xi
\end{aligned}
$$

and

$$
\begin{aligned}
S^{n}(t)= & \frac{1}{4} \int_{\mathbb{R}^{3}}\left[\left|\mathbf{e}_{n} \cdot \hat{\mathbf{u}}_{1}\right|^{2}+r^{2} v_{n}^{2}\left|\mathbf{e}_{n} \cdot \hat{\mathbf{u}}_{0}\right|^{2}\right] d^{3} \xi \\
& -\frac{1}{4} \int_{\mathbb{R}^{3}}\left[\left|\mathbf{e}_{n} \cdot \hat{\mathbf{u}}_{1}\right|^{2}-r^{2} v_{n}^{2}\left|\mathbf{e}_{n} \cdot \hat{\mathbf{u}}_{0}\right|^{2}\right] \cos \left(2 \operatorname{tr} v_{n}\right) d^{3} \xi \\
& +\frac{1}{2} \int_{\mathbb{R}^{3} 3} \operatorname{Re}\left[r v_{n}\left(\mathbf{e}_{n} \cdot \hat{\mathbf{u}}_{0}\right)\left(\mathbf{e}_{n} \cdot \hat{\mathbf{u}}_{1}\right)^{*}\right] \sin \left(2 \operatorname{tr} v_{n}\right) d^{3} \xi .
\end{aligned}
$$

The first integral in the right side of (56) and (57) does not depend on $t$. The second and third integral in (56) and (57) do depend on $t$ through the cosine and the sine factors respectively. Since $\mathbf{u}_{0} \in\left[H^{1}\left(\mathbb{R}^{3}\right)\right]^{3}, \mathbf{u}_{1} \in\left[L^{2}\left(\mathbb{R}^{3}\right)\right]^{3}$ it follows that $\left|\mathbf{e}_{n} \cdot \hat{\mathbf{u}}_{1}\right|^{2}, r^{2}\left|\mathbf{e}_{n} \cdot \hat{\mathbf{u}}_{0}\right|^{2}$, and $r\left(\mathbf{e}_{n} \cdot \hat{\mathbf{u}}_{0}\right)\left(\mathbf{e}_{n} \cdot \hat{\mathbf{u}}_{1}\right)^{*}$ are absolutely integrable functions.

By taking the limit of (56) and (57) and applying the generalized Riemann-Lebesgue Lemma 2 we obtain (48) and from these (49). This completes the proof of the theorem.

We remark that Eq. (48) establishes the asymptotic equipartition of kinetic and strain energy for each wave separately and Eq. (49) establishes the same result for the corresponding total energies.

\section{REFERENCES}

[1] D. G. Costa, On partition of energy for uniformly propagative systems, J.M.A.A. 58, 56-62 (1977)

[2] G. Dassios, Equipartition of energy for Maxwell's equations, Quart. Appl. Math. 37, 465-469 (1980)

[3] G. Dassios, Equipartition of energy in elastic wave propagation, Mechanics Research Communications (to appear)

[4] G. F. D. Duff, The Cauchy problem for elastic waves in an anisotropic medium, Phil. Mag. Roy. Soc. A252, 249-273 (1960)

[5] R. S. Duffin, Equipartition of energy in wave motion, J.M.A.A. 32, 386-391 (1970)

[6] J. A. Goldstein, An asymptotic property of solutions of wave equations, Proc. A.M.S. 23, 359-363 (1969)

[7] J. A. Goldstein, An asymptotic property of solutions of wave equations II, J.M.A.A. 32, 392-399 (1970)

[8] P. D. Lax and R. S. Phillips, Scattering theory, Academic Press, New York (1967)

[9] H. A. Levine, An equipartition of energy theorem for weak solutions of evolutionary equations in Hilbert space: the Lagrange identity method, J. Diff. Eq. 24, 197-210 (1977)

[10] M. J. P. Musgrave, Elastic waves in anisotropic media, in Progress in Solid Mechanics II, ed. Sneddon and Hill, North-Holland, New York (1960)

[11] J. L. Synge, Elastic waves in anisotropic media, J. Math. and Phys. 35, 323-334 (1956)

[12] J. L. Synge, Flux and energy for elastic waves in anisotropic media, Proc. Roy. Irish. Acad. 58, 13-21 (1956) 